\title{
Caracterização florística, fitossociológica e fenológica de trepadeiras de mata ciliar da Fazenda Campininha, Mogi Guaçu, SP, Brasil
}

\author{
Sergio Romaniuc Neto ${ }^{1,2}$, José Vanderlei Godoi ${ }^{1}$, Berta Lúcia Pereira Villagra ${ }^{1}$, Renata Jimenez de \\ Almeida-Scabbia ${ }^{1}$ e Maria Margarida da Rocha Fiuza de Melo ${ }^{1}$
}

Recebido: 20.05.2011; aceito: 16.03.2012

\begin{abstract}
Floristic, phytosociological and phenology of climbers riparian of "Fazenda Campininha", Mogi Guaçu, São Paulo State, Brazil). This study aimed to inventory component of climbing plants by studying floristic, phytosociological and phenology on a stretch of riparian vegetation of the Estação Ecológica of Fazenda Campininha, Mogi Guaçu, São Paulo State, Brazil $\left(22^{\circ} 10^{\prime} 43^{\prime \prime}-22^{\circ} 18^{\prime} 19^{\prime \prime S}\right.$ e $\left.47^{\circ} 08^{\prime} 05^{\prime \prime}-47^{\circ} 11^{\prime} 34^{\prime \prime} \mathrm{W}\right)$. In a floristic study were collected from 89 species belonging to 54 genera within 25 families. The quantitative study sampled 43 species with the highest values of importance to Adenocalymma bracteatum, Forsteronia australis and Banisteriopsis nummifera. The woody climbers accounted for $65.1 \%$ of total species and herbaceous plants, $34.8 \%$, both with fruit senescence in the middle of the dry season (June). Adaptation twining was the most frequent, occurring in $42.7 \%$ of the species. The choice of sample area and the inclusion diameter $\geq 0,2 \mathrm{~cm}$ above the soil were important to indicate that although the sample size was reduced significantly to show the diversity of species $\left(\mathrm{H}^{\prime}=2.72\right.$ nats ind $\left.^{-1}\right)$ and can be used in the indication of disturbed or preserved areas.

Key words: lianas, Mogi Guaçu, riparian forest
\end{abstract}

RESUMO - (Caracterização florística, fitossociológica e fenológica de trepadeiras de mata ciliar da Fazenda Campininha, Mogi Guaçu, SP, Brasil). O presente trabalho objetivou inventariar o componente de trepadeiras por meio de estudos florísticos, fitossociológicos e aspectos fenológicos em trecho de Mata Ciliar na Estação Ecológica da Fazenda Campininha de Mogi Guaçu ( $22^{\circ} 10^{\prime} 43^{\prime \prime}-22^{\circ} 18^{\prime} 19^{\prime \prime S}$ e $\left.47^{\circ} 08^{\prime} 05^{\prime}{ }^{\prime}-47^{\circ} 11^{\prime} 34^{\prime \prime W}\right)$. No estudo florístico foram coletadas 89 espécies, distribuídas em 54 gêneros, pertencentes a 25 famílias. O estudo quantitativo amostrou 43 espécies com os maiores valores de importância para Adenocalymma bracteatum, Forsteronia australis e Banisteriopsis nummifera. As trepadeiras lenhosas representaram $65,1 \%$ do total de espécies amostradas e as herbáceas, $34,8 \%$, ambas com senescência dos frutos mais acentuada no meio da estação seca (junho). A adaptação volúvel foi a mais frequente, ocorrendo em $42,7 \%$ das espécies. A escolha da área amostral e do diâmetro à altura da base, maior ou igual a $0,2 \mathrm{~cm}$ foram importantes para indicar que o tamanho da amostragem, embora reduzido, foi significativo para mostrar a diversidade de espécies $\left(H^{\prime}=2,72\right.$ nats ind $\left.^{-1}\right)$ e podem ser usadas na indicação de áreas perturbadas ou conservadas.

Palavras-chave: lianas, mata ripária, Mogi Guaçu

\section{Introdução}

A alta diversidade de plantas de hábito trepador em florestas tropicais, particularmente aquelas fragmentadas ou perturbadas, tem chamado a atenção por constituírem um componente que contribui significativamente na composição e estrutura das sinúsias florestais (Barros et al. 2009, Santos et al. 2009, Rubim et al. 2010).

O conhecimento das trepadeiras na estrutura das florestas tropicais avançou satisfatoriamente na última década, mas ainda há uma necessidade de obter-se mais informações sobre sua importância ecológica nos biomas brasileiros (Gentry 1982, 1985, Putz 1984, Whitmore 1990, Putz \& Mooney 1991, Villagra \& Romaniuc Neto 2010). No Brasil, os trabalhos para essa forma de vida abordam, principalmente, estudos florísticos (Morellato \& Leitão Filho 1996, Udulutsch et al. 2004, Rezende \& Ranga 2005, Tibiriçá et al. 2006, Santos et al. 2009, Villagra \& Romaniuc Neto 2010) e, com menor frequência, estudos ecológicos (Lombardi et al. 1999, Hora \& Soares 2002).

1. Instituto de Botânica, Centro de Pesquisa em Plantas Vasculares, Núcleo de Pesquisa Curadoria do Herbário, Caixa Postal 68041, 04045-972 São Paulo SP, Brasil

2. Autor para correspondência: sromaniuc@gmail.com 
No Brasil, a fenologia de espécies de trepadeiras tem sido particularmente pouco estudada. No Sudeste, estudos realizados em florestas estacionais (Morellato et al. 1989, 1990, Morellato \& Leitão Filho 1990, 1996, Rubim et al. 2010) e remanescentes de florestas ombrófilas (Rossi 1994, Penhalber 1995, Ferraz et al. 1999) detectaram que o período de maior floração ocorre na transição entre as épocas seca e úmida, de setembro a novembro. Entretanto, a frutificação depende das características morfológicas dos frutos e da síndrome de dispersão das espécies, bem como do estrato florestal ocupado pelos indivíduos adultos (Morellato \& Leitão Filho 1990). Outro fator relevante refere-se ao grupo ecológico ao qual pertence a espécie, que pode determinar sua estratégia reprodutiva.

Os trabalhos sobre a sinúsia de trepadeiras ocorrentes em Mata Ciliar, relacionando dados florísticos, fitossociológicos e fenológicos não são comuns (Oliveira \& Moreira 1992, Carmo \& Morellato 2000, Funch et al. 2002, Reys et al. 2005), provavelmente pela falta de conhecimento sobre sua importância econômica, ou ainda pela dificuldade de coleta e obtenção do material fértil, associado aos problemas metodológicos para esse grupo (Villagra \& Romaniuc Neto 2010).

O presente trabalho buscou responder as seguintes questões sobre a comunidade de trepadeiras na Mata Ciliar da Fazenda Campininha: 1) qual é a composição florística e estrutural dessa comunidade? e 2) que padrões fenológicos são encontrados para as espécies ocorrentes?

\section{Material e Métodos}

A Fazenda Campininha ocupa uma área de 470 ha no município de Mogi Guaçu, no Estado de São Paulo (22 $10^{\circ} 43^{\prime \prime}-22^{\circ} 18^{\prime} 19^{\prime \prime}$ 'S e $47^{\circ} 08^{\prime} 05^{\prime \prime}$ 47 11 '34" W), sendo formada por uma Reserva Biológica e duas Estações, uma Ecológica e outra Experimental. A vegetação é predominantemente de Cerrado, com variações de cerradão a campo, com áreas de cultivo experimental de Pinus, possuindo ao sul matas ciliares, que pode ser caracterizada, segundo Rodrigues \& Leitão Filho (2001), como uma formação ribeirinha sem influência do rio Mogi Guaçu (figura 1).

A área de estudo enquadra-se no clima Cwa de Köeppen (clima quente e úmido, com inverno seco), temperatura do mês mais frio inferior a $18^{\circ} \mathrm{C}$ e máxima do mês mais quente $22^{\circ} \mathrm{C}$ (CBH-Mogi 1999), altitude média de $600 \mathrm{~m}$ e solo do tipo Latossolo VermelhoAmarelo (Mantovani \& Martins 1988) (figura 2).
Neste trabalho foram consideradas trepadeiras todas as plantas, herbáceas ou lenhosas, que necessitam de um suporte (forófito) para se desenvolver e utilizam adaptações de escalada para ascender, incluindo a volubilidade do caule, presença de raízes preênseis ou modificações foliares, (Hegarty 1991, Villagra \& Romaniuc Neto 2010).

O levantamento florístico foi realizado no período de agosto/1991 a março/1993, por meio de coletas mensais de todos os indivíduos com flores e/ou frutos, tanto no interior das parcelas como ao longo da área de mata ciliar da Reserva Experimental. Também foram obtidas amostras de lenho para auxiliar na identificação das espécies. O material botânico foi processado conforme recomendações de Fidalgo \& Bononi (1984) e incorporado ao acervo do Herbário do Estado "Maria Eneyda P. Kauffman Fidalgo" (SP). Para a listagem das famílias adotou-se APG III (2009) e Brummitt \& Powell (1992) para a abreviação dos nomes dos autores.

Para as fenofases observadas registraram-se a ocorrência e duração do evento. Foram considerados os seguintes períodos: inicial de floração, quando as trepadeiras apresentaram as primeiras flores em antese (flores abertas); final de floração, na antese das últimas flores; inicial de frutificação, quando apareceram os primeiros frutos; e final de frutificação, quando os frutos estavam prontos para serem dispersos.

As trepadeiras foram classificadas, quanto à forma de apoio ao suporte, segundo Hegarty (1991) em: volúveis, que utilizam o caule, ramos ou pecíolos para se enrolarem no suporte; preênseis, que possuem gavinhas ou raízes adaptadas para se prender ao suporte; e escandentes, que devido à flexibilidade dos ramos crescem se apoiando na vegetação circundante, sendo frequentemente armadas de acúleos para evitar a queda.

Para o estudo quantitativo das trepadeiras, amostrou-se 0,09 ha distribuídos em 30 parcelas de $3 \times 10 \mathrm{~m}$, em faixas contíguas e paralelas ao rio na tentativa de identificar a preferência das espécies pela borda, sendo A a faixa mais próxima ao rio, $\mathrm{B}$ a intermediária e $\mathrm{C}$ mais distante do rio (figura 1). Em cada parcela foram amostradas todas as trepadeiras, que tiveram seus diâmetros medidos com paquímetro a altura da base $(\mathrm{DAB}) \geq 0,2 \mathrm{~cm}$, considerando-se cada indivíduo a partir de seu ponto de brotação do solo.

Com o auxílio do programa FITOPAC (Shepherd 1995) foram calculados os descritores quantitativos de densidade, frequência e dominância, relativas e absolutas, para cada espécie, segundo Mueller-Dombois \& Ellenberg (1974), e os índices do valor de 


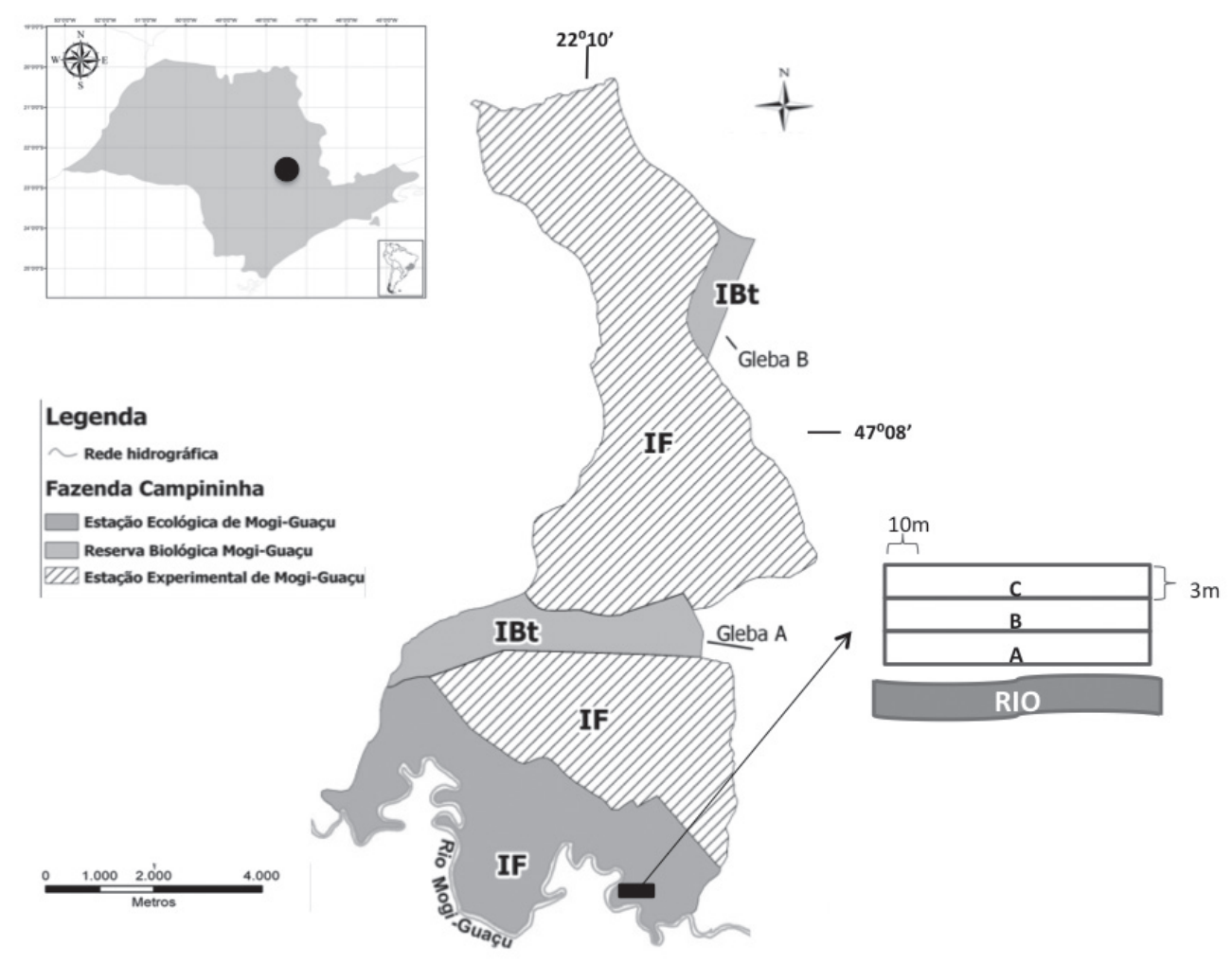

Figura 1. Localização da área de estudo alocadas na Estação Ecológica da Fazenda Campininha, Mogi Guaçu, SP, Brasil.

Figure 1. Location of studied areas in the Ecological Station of Fazenda Campininha, Mogi Guaçu, São Paulo State, Brazil.

importância (IVI) e do valor de cobertura (IVC), segundo Matteucci \& Colma (1982).

Para a análise da diversidade florística da área estudada, foi utilizado o índice de diversidade de Shannon (H'), segundo Magurran (1996).

\section{Resultados e Discussão}

No levantamento florístico foram identificadas 89 espécies de trepadeiras, distribuídas em 56 gêneros e 25 famílias (tabela 1). As famílias com maior riqueza específica foram: Bignoniaceae e Sapindaceae com 14 espécies cada (15\%), Convolvulaceae com 10 (11\%), Malpighiaceae com nove (10\%), Asteraceae com sete (8\%), Apocynaceae e Fabaceae com cinco cada (6\%). Essas sete famílias reúnem $71 \%$ das espécies encontradas no levantamento florístico. Entre os gêneros das trepadeiras lenhosas, destacamse Serjania (11 espécies), Arrabidaea (seis) e Banisteriopsis (cinco) com os maiores números de espécies encontradas na Mata Ciliar da Estação Ecológica da Fazenda Campininha. Esses gêneros são de ampla distribuição nos biomas paulistas (Kim 1996,
Somner 2009, Mamede 2010), entretanto ainda faltam dados sobre a diversidade de suas espécies em áreas de mata ciliar.

Quanto à forma de escalada, a adaptação volúvel está presente em $42,7 \%$ das espécies amostradas, seguida da preênsil, $40,4 \%$, e da escandente, $16,8 \%$. Valores aproximados foram encontrados nos levantamentos de Morelatto \& Leitão Filho (1998), Udulutsch et al. (2004) e Tibiriçá et al. (2006). As trepadeiras lenhosas representaram $64 \%$ das espécies e as herbáceas $36 \%$. Estes dados sugerem que a Mata Ciliar da Fazenda Campininha possui características de florestas maduras, corroborando com as afirmações de Putz \& Chai (1987) e Dewalt et al. (2000), que apontam um predomínio da adaptação volúvel para as florestas tropicais mais conservadas.

A área estudada está sob influência de duas estações bem definidas, a estação seca, que ocorre durante os meses de abril a setembro, e a estação chuvosa que abrange os meses de outubro a março (figura 2). A comunidade de trepadeiras floresceu e frutificou em períodos distintos, a floração ocorreu em três momentos principais: na transição da estação 
Município de Mogi Guaçu, SP, Brasil

Latitude: $22^{\circ} 13^{\prime} 00^{\prime \prime} \quad$ Longitude: $46^{\circ} 33^{\prime} 00^{\prime \prime} \quad$ Altitude média: $640 \mathrm{~m}$

Municipio de Mogi Guaçu, SP, Brasil

Latitude: $22^{\circ} 13^{\prime} 00^{\prime \prime} \quad$ Longitude: $46^{\circ} 33^{\prime} 00^{\prime \prime} \quad$ Altitude média: $640 \mathrm{~m}$

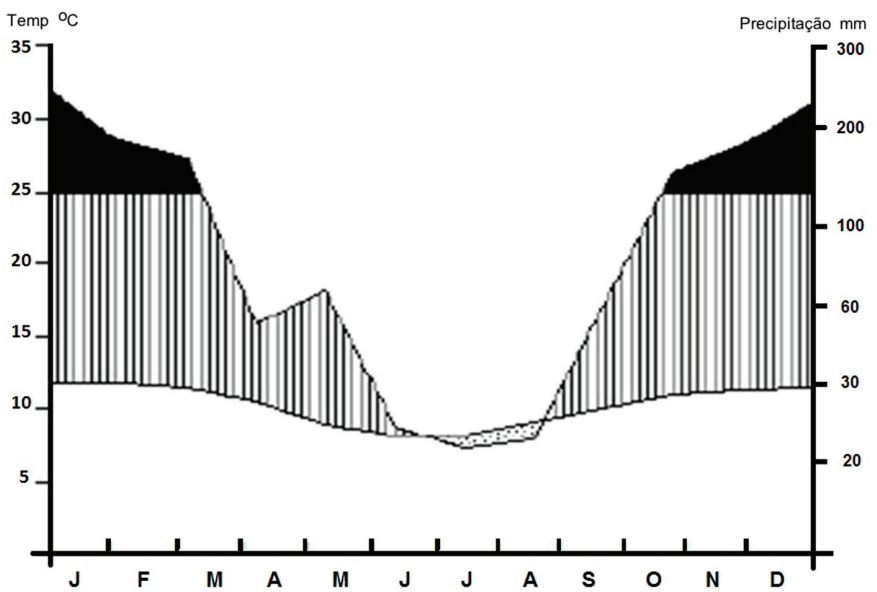

Figura 2. Diagrama climático para a região do município de Mogi Guaçu, SP, Brasil, no período de 1971 a 2005. A curva inferior mostra a temperatura média mensal total (intervalos de $20 \mathrm{~mm}$ no eixo Y), exceto para a zona preta que representa intervalos super úmidos com precipitação superior a $100 \mathrm{~mm}$ por mês, quando a escala é reduzida a 1/10. Zona com traços: período úmido; zona pontilhada: período seco. Acima, no gráfico, são mostradas a altitude, temperatura média anual $\left({ }^{\circ} \mathrm{C}\right)$ e precipitação (mm). Fonte: CEPAGRI - Centro de Pesquisas Meteorológicas e Climáticas Aplicadas à Agricultura, Cidade Universitária "Zeferino Vaz" - Campinas, SP, Brasil. http://www.cpa.unicamp.br/outras-informacoes/clima_muni_347.html (acesso em 15.08.2011).

Figure 2. Climate diagram for the region of Mogi Guaçu, São Paulo State, Brazil, in the period 1971 to 2005. The lower curve shows the total monthly average temperature (20 mm intervals on the Y axis), except for the black zone represents intervals super humid with rainfall exceeding $100 \mathrm{~mm}$ per month, when the scale is reduced to 1/10. Area with traits: the wet season; dotted area: a dry season. Above the chart are shown the altitude, mean annual temperature $\left({ }^{\circ} \mathrm{C}\right)$ and precipitation $(\mathrm{mm})$. Source: CEPAGRI - Centro de Pesquisas Meteorológicas e Climáticas Aplicadas à Agricultura, Cidade Universitária "Zeferino Vaz" - Campinas, Sao Paulo State, Brazil. http://www.cpa.unicamp.br/outras-informacoes/clima_muni_347.html (acesso em 15.08.2011).

seca-úmida (março-abril), no meio (junho) e final (setembro) da estação seca (figura 3). A frutificação nas trepadeiras herbáceas foi predominantemente no meio da estação seca (junho), enquanto para as trepadeiras lenhosas foi distribuída ao longo do ano, com aumento também em junho (figura 4).

Os dados de senescência dos frutos, mais acentuada no meio da estação seca, tanto para as trepadeiras lenhosas quanto para as herbáceas, são similares aos registrados por Gentry (1991) e por Morellato \& Leitão Filho (1996). Esse fato pode estar relacionado à estratégia de reprodução das trepadeiras, favorecendo a dispersão de frutos e sementes por animais ao aumentar a oferta de alimento em épocas desfavoráveis (Opler et al. 1991).

Do total de espécies observadas em campo, 67 foram visitadas por animais, sendo $95,5 \%$ por insetos e somente $4,5 \%$ por aves. Morelatto \& Leitão Filho (1996) afirmaram que 65\% das espécies de trepadeiras lenhosas são polinizadas por abelhas e em menor quantidade por outros insetos pequenos. Entretanto, quanto às síndromes de dispersão, a comunidade de trepadeiras da Mata Ciliar da Fazenda Campininha, possui dispersão por anemocoria (75,2\%). Malpighiaceae, Bignoniaceae e Sapindaceae, que apresentam maior diversidade específica, são predominantemente anemocóricas, facilitada pela morfologia de seus frutos ou sementes alados.

No estudo fitossociológico da comunidade de trepadeiras foram amostrados 862 indivíduos em uma área de 0,09 ha, distribuídos em 43 espécies (tabela 2), pertencentes a 16 famílias. A alta densidade encontrada é similar a mencionada por Proctor et al. (1983) para florestas em solos aluviais.

A diversidade obtida de $\mathrm{H}^{\prime}=2,725$ nats ind $^{-1}$, revelou maior riqueza de espécies comparada aos demais levantamentos até então desenvolvidos para o hábito trepador (Citadini-Zanette et al. 1997, Lima et al. 1997, Venturi 2000, Hora \& Soares 2002, Villagra \& Romaniuc Neto 2010). As famílias que mais contribuíram em número de espécies foram Sapindaceae (oito), Malpighiaceae (oito) e Apocynaceae (seis). Bignoniaceae (quatro) foi a quarta família mais importante em número de espécies e a 
Tabela 1. Trepadeiras da mata ciliar da Fazenda Campininha, Mogi Guaçu, SP, Brasil. Propriedade morfológica: herbácea $(\mathrm{H})$, lenhosa (L). Forma de escalada : volúvel (Vol), escandente (Esc), preênsil (Pre).

Table 1. Climbers riparian of Fazenda Campininha Biological Reserva and Experimental Station of Mogi Guaçu, São Paulo State, Brazil. Morphological property: herbaceous(H), woody (L). Form of climbing twining(Vol), scrambling(Esc), tendril climbing(Pre).

\begin{tabular}{llll}
\hline Espécie & $\begin{array}{l}\text { Propriedade Forma de } \\
\text { morfológica escalada }\end{array}$ & Material testemunho & $\begin{array}{c}\text { Síndrome } \\
\text { de dispersão }\end{array}$ \\
\hline
\end{tabular}

Acanthaceae

Mendoncia velloziana Mart.

Amaranthaceae

Chamissoa altissima (Jacq.) Kunth

Hebanthe eriantha (Poir.) Pedersen

Apocynaceae

Condylocarpon isthmicum (Vell.) A.DC.

Forsteronia pubescens A.DC.

Oxypetalum appendiculatum Mart.

Prestonia coalita (Vell.) Woodson

Prestonia riedelii (Müll.Arg.) Markgr.

Aristolochiaceae

Aristolochia galeata Mart. \& Zucc.

Asteraceae

Austrocritonia velutina (Gardner) R.M.King \& H.Rob.

Bidens segetum Mart. ex Colla

Dasyphyllum vagans (Gardner) Cabrera

Mikania cordifolia (L.f.) Willd.

Mikania salviifolia Gardner

Mikania involucrata Hook. \& Arn.

Stomatanthes dictyophyllus (DC.) R.M.King \& H.Rob.

Bignoniaceae

Adenocalymma bracteatum (Cham.) DC.

Adenocalymma paulistarum Bureau \& K.Schum

Anemopaegma chamberlaynii (Sims) Bureau \& K.Schum

Arrabidaea brachypoda (DC.) Bureau

Arrabidaea pubescens (L.) A.H.Gentry

Arrabidaea pulchra (Cham.) Sandwith

Arrabidaea pulchella (Cham.) Bureau

Arrabidaea samydoides (Cham.) Sandwith

Arrabidaea triplinervia (Mart. ex DC.) Baill.

Clytostoma binatum (Thunb.) Sandwith

Cuspidaria floribunda (DC.) A.H.Gentry

Distictella elongata (Vahl.) Urb.

Lundia obliqua Sond.
$\mathrm{H}$

L Esc

$\mathrm{H} \quad$ Esc

L

L

$\mathrm{H}$

L

L

$\mathrm{H}$

$\mathrm{H}$

$\mathrm{H}$

$\mathrm{L}$

$\mathrm{H}$

$\mathrm{H}$

$\mathrm{H}$

$\mathrm{H}$

L

L

L

L

L

L

L

L

L

L

L

L

L

Vol

Vol

Vol

Vol

Vol

Vol

Vol

Vol

Vol

Esc

Vol

Vol

Vol

Esc

Pre

Pre

Pre

Pre
J.V. Godoi \& S. Romaniuc Neto 163

S. Romaniuc Neto et. al. 1316

J.V. Godoi et al. 245

$J$

J.V. Godoi et. al. 152

J.V. Godoi \& D.F. Pereira 263

anemocoria

J.V. Godoi \& S. Romaniuc Neto 357

S. Romaniuc Neto \& L. Rossi 1166

C.B. Toledo et al. 512

W. Mantovani 485

J.V. Godoi (UEC 132078)

M. Kuhlmann 3929

anemocoria

anemocoria

anemocoria

anemocoria

anemocoria

anemocoria

anemocoria

S. Romaniuc Neto \& J.V. Godoi 1295 anemocoria

S. Romaniuc Neto \& R. Zifirino 1269 anemocoria

J.V. Godoi 229

anemocoria

J. Mattos 13685

S. Romaniuc Neto (UEC 127171)

anemocoria

J.V. Godoi \& S. Romaniuc Neto 195

anemocoria

M. Kirizawa \& W. Mantovani 1313

J.V. Godoi \& S. Romaniuc Neto 162

J.V. Godoi et al. 289

anemocoria

anemocoria

anemocoria

J.V. Godoi \& S. Romaniuc Neto 272

J.V. Godoi (UEC 127209)

anemocoria

anemocoria

S. Romaniuc Neto \& L. Rossi 1179

J.V. Godoi et al. 292 anemocoria

anemocoria

anemocoria 
Tabela 1 (continuação)

\begin{tabular}{|c|c|c|c|c|}
\hline Espécie & $\begin{array}{l}\text { Propriedade } \\
\text { morfológica }\end{array}$ & $\begin{array}{r}\text { Forma de } \\
\text { escalada }\end{array}$ & Material examinado & $\begin{array}{c}\text { Síndrome } \\
\text { de dispersão }\end{array}$ \\
\hline Pyrostegia venusta (Ker Gawl.) Miers & $\mathrm{L}$ & Pre & S. Romaniuc Neto \& L. Rossi 1175 & anemocoria \\
\hline \multicolumn{5}{|l|}{ Celastraceae } \\
\hline Hippocratea volubilis L. & $\mathrm{L}$ & Vol & J.V. Godoi et al 201 & anemocoria \\
\hline \multicolumn{5}{|l|}{ Convolvulaceae } \\
\hline Aniseia martinicensis (Jacq.) Choisy & $\mathrm{H}$ & Vol & J.V. Godoi \& D.F. Pereira 179 & anemocoria \\
\hline Ipomoea alba $\mathrm{L}$. & $\mathrm{H}$ & Vol & L. Rossi et al. 856 & anemocoria \\
\hline Ipomoea cairica (L.) Sweet. & $\mathrm{H}$ & Vol & J.V. Godoi et al. 381 & anemocoria \\
\hline Ipomoea setifera Poir. & $\mathrm{H}$ & Vol & D.F. Pereira 40 & anemocoria \\
\hline Jacquemontia densiflora (Meisn.) Hallier f. & $\mathrm{H}$ & Vol & J.V. Godoi 1368 & anemocoria \\
\hline Jacquemontia velutina Choisy & $\mathrm{H}$ & Vol & J.V. Godoi et al. 215 & anemocoria \\
\hline Merremia aegyptia (L.) Urb. & $\mathrm{H}$ & Vol & J.V. Godoi et al. 372 & anemocoria \\
\hline Merremia cissoides (Lam.) Hallier f. & $\mathrm{H}$ & Vol & S. Romaniuc Neto et al. 1371 & anemocoria \\
\hline Merremia macrocalyx (Ruiz \& Pav.) O’Donell & $\mathrm{H}$ & Vol & S. Romaniuc Neto \& J.V. Godoi 1293 & anemocoria \\
\hline Merremia umbellata (L.) Hallier f. & $\mathrm{H}$ & Vol & J.V. Godoi et al. 367 & anemocoria \\
\hline \multicolumn{5}{|l|}{ Cucurbitaceae } \\
\hline Momordica charantia $\mathrm{L}$. & $\mathrm{H}$ & Pre & J.V. Godoi et al. 286 & zoocoria \\
\hline \multicolumn{5}{|l|}{ Dilleniaceae } \\
\hline Davilla rugosa Poir. & $\mathrm{L}$ & Esc & J.V. Godoi \& D.F. Pereira 266 & zoocoria \\
\hline Doliocarpus dentatus (Aubl.) Standl. & $\mathrm{L}$ & Esc & E. Forero et al. 8454 & zoocoria \\
\hline \multicolumn{5}{|l|}{ Euphorbiaceae } \\
\hline Dalechampia micromeria Baill. & $\mathrm{H}$ & Vol & J.V. Godoi \& S. Romaniuc Neto 275 & autocoria \\
\hline \multicolumn{5}{|l|}{ Fabaceae } \\
\hline Centrosema angustifolium (Kunth) Benth. & $\mathrm{L}$ & Esc & S.A.C. Chiea \& M.M.R.F. Melo 82 & autocoria \\
\hline Dalbergia frutescens (Vell.) Britton & $\mathrm{L}$ & Vol & H.F. Leitão-Filho \& K. Yamamoto 6031 & anemocoria \\
\hline Dioclea rufescens Benth. & $\mathrm{L}$ & Esc & Tamashiro et al. 6521 & autocoria \\
\hline Senegalia polyphylla (DC.) Britton \& Rose & $\mathrm{L}$ & Esc & S. Romaniuc Neto et al. 1098 & autocoria \\
\hline Senegalia tenuifolia (L.) Britton \& Rose & $\mathrm{L}$ & Esc & S. Romaniuc Neto et al 1108 & autocoria \\
\hline \multicolumn{5}{|l|}{ Malpighiaceae } \\
\hline Banisteriopsis argyrophylla (A. Juss.) B.Gates & $\mathrm{L}$ & Vol & J. Mattos 8987 & anemocoria \\
\hline $\begin{array}{l}\text { Banisteriopsis malifolia (Ness. \& Mart.) B. } \\
\text { Gates var. malifolia }\end{array}$ & $\mathrm{L}$ & Vol & S.A.C. Chiea \& M.M.R.F. Melo 51 & anemocoria \\
\hline $\begin{array}{l}\text { Diplopterys pubipetala (A. Juss.) W.R. } \\
\text { Anderson \& C.C.Davis }\end{array}$ & $\mathrm{L}$ & Vol & E. Forero et al. 8236 & anemocoria \\
\hline Banisteriopsis nummifera (A. Juss.) B. Gates & $\mathrm{L}$ & Vol & S. Romaniuc Neto et al. 1104 & anemocoria \\
\hline Banisteriopsis variabilis B. Gates & $\mathrm{L}$ & Vol & W. Mantovani 1330 & anemocoria \\
\hline Byrsonima coccolobifolia Kunth & $\mathrm{L}$ & Vol & A. Custodio Filho 423 & anemocoria \\
\hline Heteropterys crenulata Mart. ex Griseb & $\mathrm{L}$ & Vol & S. Romaniuc Neto \& L. Rossi 1191 & anemocoria \\
\hline Heteropterys eglandulosa A. Juss. & $\mathrm{L}$ & Vol & D.F. Pereira et al. 190 & anemocoria \\
\hline Mascagnia cordifolia (A. Juss.) Griseb. & $\mathrm{L}$ & Vol & R. Zifirino \& S. Romaniuc Neto 6 & anemocoria \\
\hline \multicolumn{5}{|l|}{ Menispermaceae } \\
\hline Cissampelos andromorpha DC. & $\mathrm{H}$ & Vol & S.L. Jung et al. 96 & $\begin{array}{l}\text { zoocoria } \\
\text { continua }\end{array}$ \\
\hline
\end{tabular}


Tabela 1 (continuação)

\begin{tabular}{|c|c|c|c|c|}
\hline Espécie & $\begin{array}{l}\text { Propriedade } \\
\text { morfológica }\end{array}$ & $\begin{array}{l}\text { Forma de } \\
\text { escalada }\end{array}$ & Material testemunho & $\begin{array}{c}\text { Síndrome } \\
\text { de dispersão }\end{array}$ \\
\hline Cissampelos glaberrima A. St.-Hil. & $\mathrm{H}$ & Vol & S. Romaniuc Neto \& J.V. Godoi 1048 & zoocoria \\
\hline \multicolumn{5}{|l|}{ Nyctaginaceae } \\
\hline Pisonia aculeata L. & $\mathrm{L}$ & Esc & S. Romaniuc Neto \& R. Zifirino 1265 & zoocoria \\
\hline \multicolumn{5}{|l|}{ Passifloraceae } \\
\hline Passiflora miersii Mast. & $\mathrm{H}$ & Pre & S. Romaniuc Neto \& J.V. Godoi 1047 & zoocoria \\
\hline \multicolumn{5}{|l|}{ Phytolaccaceae } \\
\hline Seguieria americana L. & $\mathrm{L}$ & Esc & J.V. Godoi et al. 293 & anemocoria \\
\hline \multicolumn{5}{|l|}{ Polygalaceae } \\
\hline Bredemeyera floribunda Willd. & $\mathrm{L}$ & Esc & C.E.O. Lohmann et al. 9 & zoocoria \\
\hline \multicolumn{5}{|l|}{ Rhamnaceae } \\
\hline Gouania inornata Reissek & $\mathrm{L}$ & Pre & J.V. Godoi et al 54 & anemocoria \\
\hline Gouania virgata Reissek & $\mathrm{L}$ & Pre & S. Romaniuc Neto \& L. Rossi 11665 & anemocoria \\
\hline \multicolumn{5}{|l|}{ Rubiaceae } \\
\hline Chiococca alba (L.) Hitchc. & $\mathrm{H}$ & Vol & D.F. Pereira 206 & zoocoria \\
\hline Manettia cordifolia Mart. & $\mathrm{H}$ & Vol & J.V. Godoi \& S. Romaniuc Neto 29 & autocoria \\
\hline \multicolumn{5}{|l|}{ Sapindaceae } \\
\hline Cardiospermum grandiflorum Sw. & $\mathrm{L}$ & Pre & S. Romaniuc Neto \& L. Rossi 1192 & anemocoria \\
\hline Paullinia elegans Cambess. & $\mathrm{L}$ & Pre & S. Romaniuc Neto \& J.V. Godoi 1339 & zoocoria \\
\hline Serjania acoma Radlk. & $\mathrm{L}$ & Pre & J. Mattos 12279 & anemocoria \\
\hline Serjania caracasana (Jacq.) Willd. & $\mathrm{L}$ & Pre & J.V. Godoi et al. 214 & anemocoria \\
\hline Serjania erecta Radlk. & $\mathrm{L}$ & Pre & J. Mattos \& F. Silveira 28454 & anemocoria \\
\hline Serjania fuscifolia Radlk. & $\mathrm{L}$ & Pre & S. Romaniuc Neto et al. 1384 & anemocoria \\
\hline Serjania laruotteana Cambess. & $\mathrm{L}$ & Pre & J.V. Godoi et al 363 & anemocoria \\
\hline Serjania lethalis A.St.-Hil. & $\mathrm{L}$ & Pre & J.V. Godoi \& S. Romaniuc Neto 237 & anemocoria \\
\hline Serjania mansiana Mart. & $\mathrm{L}$ & Pre & C.E.O. Lohmann et al. 19 & anemocoria \\
\hline Serjania meridionalis Cambess. & $\mathrm{L}$ & Pre & J.V. Godoi \& S. Romaniuc Neto 192 & anemocoria \\
\hline Serjania multiflora Cambess. & $\mathrm{L}$ & Pre & J.V. Godoi et al. 210 & anemocoria \\
\hline Serjania ovalifolia Radlk. & $\mathrm{L}$ & Pre & W. Mantovani 1609 & anemocoria \\
\hline Serjania tripleuria Ferrucci & $\mathrm{L}$ & Pre & J.V. Godoi \& S. Romaniuc Neto 200 & anemocoria \\
\hline Urvillea ulmacea Kunth & $\mathrm{L}$ & Pre & S. Romaniuc Neto et al. 1324 & anemocoria \\
\hline \multicolumn{5}{|l|}{ Smilacaceae } \\
\hline Smilax campestris Griseb. & $\mathrm{H}$ & Pre & J.V. Godoi et al 216 & zoocoria \\
\hline Smilax fluminensis Steud. & $\mathrm{H}$ & Pre & S. Romaniuc Neto \& R. Zifirino 1260 & zoocoria \\
\hline Smilax quinquenervia Vell. & $\mathrm{H}$ & Pre & S. Romaniuc Neto \& R. Zifirino 1257 & zoocoria \\
\hline \multicolumn{5}{|l|}{ Trigoniaceae } \\
\hline Trigonia nivea Cambess. & $\mathrm{H}$ & Esc & J.V. Godoi \& D.F. Pereira 170 & anemocoria \\
\hline \multicolumn{5}{|l|}{ Violaceae } \\
\hline Anchietea pyrifolia (Mart.) G. Don & $\mathrm{L}$ & Esc & R. Zifirino \& S. Romaniuc Neto 7 & anemocoria \\
\hline \multicolumn{5}{|l|}{ Vitaceae } \\
\hline Cissus erosa Rich. & $\mathrm{H}$ & Pre & M.M.R.F. Melo \& S.A.C. Chiea 174 & zoocoria \\
\hline
\end{tabular}


Tabela 2. Descritores quantitativos para as espécies de trepadeiras amostradas em trecho de mata ciliar na Reserva Biológica e Estação Experimental de Mogi Guaçu, Mogi Guaçu, SP, Brasil. Ni: número de indivíduos amostrados; No: número de parcelas em que a espécie ocorreu; $\mathrm{AB}$ : área basal por hectare $\left(\mathrm{m}^{2}\right)$; DR: densidade relativa (\%); DoR: dominância relativa $(\%)$; FR: frequência relativa (\%); IVI: índice do valor de importância; IVC: índice do valor de cobertura.

Table 2. Quantitative descriptors for species sampled from climbing plants stretch of riparian forest in the Biological Reserve and Experimental Station of Mogi Guaçu, Mogi Guaçu, São Paulo State, Brazil. Ni: number of individuals sampled; No: number of plots where the species occurred; AB: basal area per hectare (square meters); DR: relative density (\%) DoR: the relative dominance (\%); FR: relative frequency (\%); IVI: importance value index; IVC: index of the value of coverage.

\begin{tabular}{|c|c|c|c|c|c|c|c|c|}
\hline Espécie & $\mathrm{Ni}$ & No & $\mathrm{AB}$ & $\mathrm{DR}$ & DoR & FR & IVI & IVC \\
\hline Adenocalymma bracteatum & 163 & 29 & 0,0693 & 18,87 & 17,14 & 10,98 & 46,99 & 36,01 \\
\hline Forsteronia australis & 227 & 25 & 0,0371 & 26,27 & 9,17 & 9,47 & 44,92 & 35,45 \\
\hline Banisteriopsis nummifera & 52 & 15 & 0,1271 & 6,02 & 31,40 & 5,68 & 43,10 & 37,42 \\
\hline Hippocratea volubilis & 41 & 20 & 0,0261 & 4,75 & 6,44 & 7,58 & 18,76 & 11,19 \\
\hline Forsteronia thyrsoidea & 43 & 8 & 0,0261 & 4,98 & 6,44 & 3,03 & 14,45 & 11,42 \\
\hline Serjania communis & 34 & 14 & 0,0046 & 3,94 & 1,14 & 5,30 & 10,37 & 5,07 \\
\hline Paullinia elegans & 27 & 10 & 0,0080 & 3,13 & 1,98 & 3,79 & 8,89 & 5,10 \\
\hline Paullinia micrantha & 34 & 9 & 0,0061 & 3,94 & 1,51 & 3,41 & 8,85 & 5,44 \\
\hline Clytostoma binatum & 20 & 10 & 0,0110 & 2,31 & 2,71 & 3,79 & 8,81 & 5,02 \\
\hline Prestonia coalita & 30 & 11 & 0,0034 & 3,47 & 0,84 & 4,17 & 8,48 & 4,31 \\
\hline Seguieria americana & 4 & 3 & 0,0242 & 0,46 & 5,99 & 1,14 & 7,59 & 6,46 \\
\hline Malpighiaceae sp. 1 & 16 & 7 & 0,0109 & 1,85 & 2,70 & 2,65 & 7,20 & 4,55 \\
\hline Machaerium lanceolatum & 17 & 11 & 0,0037 & 1,97 & 0,91 & 4,17 & 7,04 & 2,88 \\
\hline Chamissoa altissima & 13 & 9 & 0,0079 & 1,50 & 1,94 & 3,41 & 6,86 & 3,45 \\
\hline Pisonia aculeata & 10 & 7 & 0,0094 & 1,16 & 2,32 & 2,65 & 6,13 & 3,48 \\
\hline Dioclea rufescens & 10 & 4 & 0,0119 & 1,16 & 2,95 & 1,52 & 5,62 & 4,11 \\
\hline Heteropterys sp. 1 & 26 & 6 & 0,0006 & 3,01 & 0,16 & 2,27 & 5,44 & 3,17 \\
\hline Serjania laruotteana & 12 & 7 & 0,0024 & 1,39 & 0,59 & 2,65 & 4,63 & 1,98 \\
\hline Malpighiaceae sp. 2 & 10 & 6 & 0,0013 & 1,16 & 0,33 & 2,27 & 3,76 & 1,49 \\
\hline Dioscorea multiflora & 12 & 4 & 0,0013 & 1,39 & 0,07 & 1,52 & 2,98 & 1,46 \\
\hline Malpighiaceae sp. 3 & 6 & 4 & 0,0012 & 0,69 & 0,29 & 1,52 & 2,50 & 0,98 \\
\hline Condylocarpum isthmicum & 5 & 3 & 0,0027 & 0,58 & 0,67 & 1,14 & 2,39 & 1,25 \\
\hline Ipomoea cairica & 5 & 4 & 0,0001 & 0,58 & 0,03 & 1,52 & 2,12 & 0,61 \\
\hline Hebanthe eriantha & 5 & 4 & 0,0001 & 0,58 & 0,02 & 1,52 & 2,11 & 0,60 \\
\hline Forsteronia pilosa & 3 & 3 & 0,0014 & 0,35 & 0,33 & 1,14 & 1,82 & 0,68 \\
\hline Cardiospermum grandiflorum & 4 & 3 & 0,0007 & 0,46 & 0,17 & 1,14 & 1,77 & 0,63 \\
\hline Davilla rugosa & 4 & 3 & 0,0003 & 0,46 & 0,09 & 1,14 & 1,68 & 0,55 \\
\hline Forsteronia pubescens & 3 & 2 & 0,0001 & 0,35 & 0,03 & 0,76 & 1,13 & 0,37 \\
\hline Mikania sp. 1 & 3 & 2 & 0,0001 & 0,35 & 0,01 & 0,76 & 1,11 & 0,3 \\
\hline Gouania inornata & 2 & 2 & 0,0002 & 0,23 & 0,04 & 0,76 & 1,03 & 0,27 \\
\hline Serjania meridionalis & 2 & 2 & 0,0001 & 0,23 & 0,02 & 0,76 & 1,01 & 0,25 \\
\hline Asteraceae sp. 1 & 2 & 2 & 0,0000 & 0,23 & 0,00 & 0,76 & 0,99 & 0,23 \\
\hline Serjania fuscifolia & 2 & 2 & 0,0000 & 0,23 & 0,00 & 0,76 & 0,99 & 0,2 \\
\hline Smilax sp. 1 & 2 & 2 & 0,0000 & 0,23 & 0,00 & 0,76 & 0,99 & 0,23 \\
\hline
\end{tabular}


Tabela 2 (continuação)

\begin{tabular}{lrrrrrrrr}
\hline Espécie & $\mathrm{Ni}$ & $\mathrm{No}$ & $\mathrm{AB}$ & $\mathrm{DR}$ & DoR & FR & IVI & IVC \\
\hline Herreria salsaparilha & 5 & 1 & 0,0001 & 0,58 & 0,03 & 0,38 & 0,99 & 0,61 \\
Serjania multiflora & 1 & 1 & 0,0008 & 0,12 & 0,20 & 0,38 & 0,69 & 0,31 \\
Merremia macrocalyx & 1 & 1 & 0,0000 & 0,12 & 0,01 & 0,38 & 0,50 & 0,12 \\
Adenocalymma comosum & 1 & 1 & 0,0000 & 0,12 & 0,01 & 0,38 & 0,50 & 0,12 \\
Smilax campestris & 1 & 1 & 0,0000 & 0,12 & 0,00 & 0,38 & 0,50 & 0,12 \\
Gouania virgata & 1 & 1 & 0,0000 & 0,12 & 0,00 & 0,38 & 0,50 & 0,12 \\
Malpighiaceae sp. 4 & 1 & 1 & 0,0000 & 0,12 & 0,00 & 0,38 & 0,50 & 0,12 \\
Macfadyena unguis-cati & 1 & 1 & 0,0000 & 0,12 & 0,00 & 0,38 & 0,50 & 0,12 \\
Doliocarpus dentatus & 1 & 1 & 0,0000 & 0,12 & 0,00 & 0,38 & 0,50 & 0,12 \\
\hline
\end{tabular}

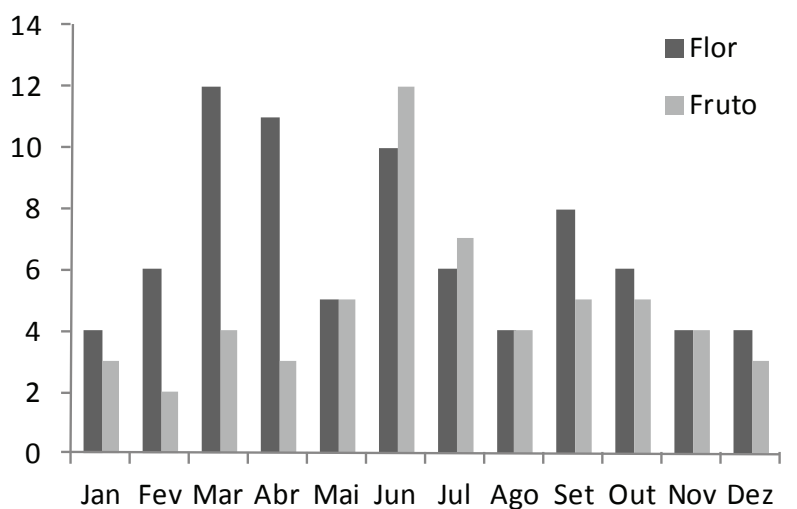

Figura 3. Período (mês) de floração e frutificação em relação ao número de espécies das trepadeiras herbáceas de mata ciliar na Estação Experimental de Mogi Guaçu, SP, Brasil.

Figure 3. Flowering and fruiting period (month) in relation to the number of herbaceous climbers of riparian forest in the Experimental Station of Mogi Guaçu, São Paulo State, Brazil.

única presente nas três faixas amostradas, tanto mais próximo quanto mais distante do rio.

As dez espécies mais abundantes representaram $78 \%$ dos indivíduos amostrados, dentre elas com maiores valores de importância foram Adenocalymma bracteatum, Forsteronia australis e Banisteriopsis nummifera, com 46,99, 44,92 e 42,10\%, respectivamente, as duas primeiras representando mais de $45 \%$ do total da amostragem, possuindo principalmente alta densidade, enquanto que Banisteriopsis nummifera possui maior dominância, com elevada área basal.

Algumas espécies, com valores altos para os índices do valor de cobertura, são representadas por poucos indivíduos com grandes diâmetros, como Seguiera americana e Pisonia aculeata, contrariamente aquelas com muitos indivíduos de pequenos diâmetros como Hippocratea volubilis e Forsteronia thyrsoidea.

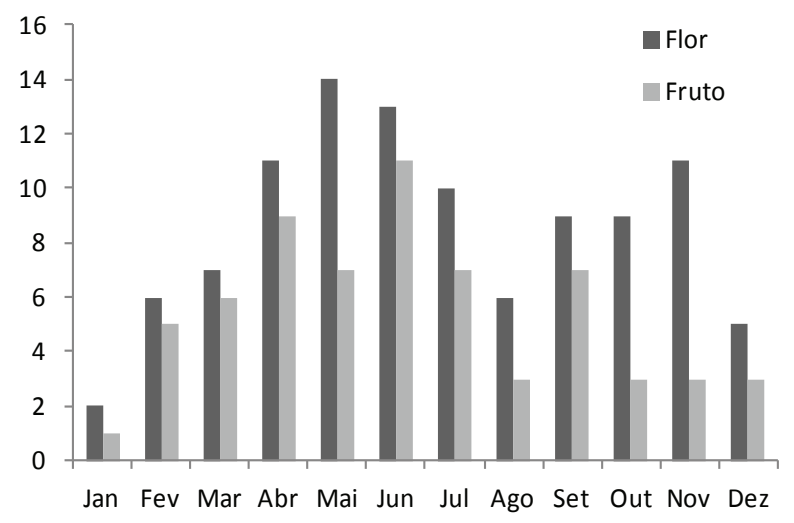

Figura 4. Período (mês) de floração e frutificação em relação ao número de espécies das trepadeiras lenhosas de mata ciliar na Estação Ecológica da Fazenda Campininha, Mogi Guaçu, SP, Brasil.

Figure 4. Flowering and fruiting period (month) in relation to the number of woody climbers of riparian forest in the Ecological Station of Fazenda Campininha, Mogi Guaçu, São Paulo State, Brazil.

Diferentemente do levantamento florístico, que obteve predominância de trepadeiras lenhosas, no quantitativo, a distribuição em classes de diâmetro revelou que $83,9 \%$ do total de indivíduos amostrados têm DAB entre 0,2-3 cm, indicando o predomínio de trepadeiras herbáceas, que participam significativamente do estágio inicial de regeneração em margens de rio.

A escolha do diâmetro na altura da base (DAB) foi experimental e resultou em problemas na medição de estolões, o que provavelmente pode superestimar o real diâmetro de algumas espécies, e assim não deve ser incentivado, visto que Schnitzer et al. (2006) propuseram em protocolo para estudos dessa sinúsia a padronização da medição dos indivíduos trepadores a 1,30 $\mathrm{m}$ do ponto central de enraizamento.

$\mathrm{Na}$ amostragem quantitativa, Sapindaceae obteve maior riqueza em número de espécies, representada 
principalmente por Serjania. Essa riqueza pode ser explicada por esse gênero possuir alta diversidade para matas ciliares, (Acevedo-Rodríguez 1990), pois pode ser disperso tanto pelo vento, como pela água corrente.

Embora, Banisteriopsis nummifera e Dioclea rufescens tenham mostrado uma maior frequência na faixa $\mathrm{A}$, mais próxima ao rio, foram encontradas também nas demais faixas. Isso demonstra que a divisão da parcela em faixas contíguas não atendeu a proposta de investigação da influência na estrutura da vegetação da proximidade ao rio, na Mata Ciliar da Fazenda Campininha.

As trepadeiras herbáceas, que são geralmente excluídas dos levantamentos fitossociológicos, apresentaram alta densidade, revelando a importância do critério de inclusão adotado, $0,2 \mathrm{~cm}$ para o diâmetro.

A comunidade de hábito trepador em matas ciliares é relativamente pouco estudada quanto aos aspectos florísticos, quantitativos e fenológicos. A complementariedade desses aspectos pode auxiliar na obtenção de dados úteis para a preservação e políticas de manejo em áreas de mata ciliar na região do rio Mogi Guaçu.

\section{Agradecimentos}

À Fundação de Amparo à Pesquisa no Estado de São Paulo - FAPESP (Processo 2174-5/91), pela bolsa concedida e ao Instituto de Botânica, Secretaria do Meio Ambiente (Processo SMA 20873/90), pelo apoio financeiro.

\section{Literatura citada}

APG. 2009. An update of the Angiosperm Phylogeny Group classification for the orders and families of flowering plants: APG III. Botanical Journal of the Linnean Society 161: 105-121.

Acevedo-Rodríguez, P. 1990. Distributional patterns in Brazilian Serjania (Sapindaceae). Acta Botanica Brasilica 4: 69-82.

Barros, A.A.M., Ribas, L.A. \& Araujo, D.S.D. 2009. Trepadeiras do Parque Estadual da Serra da Tiririca, Rio de Janeiro, Brasil. Rodriguésia 60: 681-694.

Brummitt, R.K. \& Powell, C.E. 1992. Authors of plant names. Royal Botanic Gardens, Kew.

Carmo, M.R.B. \& Morellato, L.P.C. 2000. Fenologia de árvores e arbustos das matas ciliares da Bacia do rio Tibagi, Estado do Paraná, Brasil. In: R.R. Rodrigues \& H.F. Leitão Filho (eds.). Matas Ciliares: conservação e recuperação. Edusp, São Paulo, pp. 125-141.
Citadini-Zanette, V., Soares, J.J. \& Martinello, C.M. 1997. Lianas de um remanescente florestal da microbacia do Rio Novo, Orleans, Santa Catarina, Brasil. Insula 26: 45-63.

Comitê da Bacia Hidrográfica do Rio Mogi Guaçu, CBH-Mogi. 1999. Diagnóstico da bacia hidrográfica do rio Mogi Guaçu: Relatório Zero.

Dewalt S.J., Schnitzer S.A. \& Denslow J.S. 2000. Density and diversity of lianas along a chronosequence in a central Panamanian lowland forest. Journal of Tropical Ecology 16: 1-19.

Ferraz, D.K.,Artes, R., Mantovani, W. \& Magalhães, L.M. 1999. Fenologia de árvores em fragmento de mata em São Paulo, SP. Revista Brasileira de Biologia 59: 305-317.

Fidalgo, O. \& Bononi, V.L.R. (coords.). 1984. Técnicas de coleta, preservação e herborização de material botânico. Instituto de Botânica, São Paulo.

Funch, L.S., Funch, R. \& Barroso, G.M. 2002. Phenology of gallery and montane forest in the Chapada Diamantina, Bahia, Brazil. Biotropica 34: 40-50.

Gentry, A.H. 1982. Patterns of neotropical plant species diversity. Evolutionary Biology 15: 1-84.

Gentry, A.H. 1985. An ecotaxonomic survey of Panamanian lianas. In: W.G. D’Arcy \& M. Correa (eds.). Historia Natural de Panama. Missouri Botanical Garden, St Louis, pp. 29-42.

Gentry, A.H. 1991. The distribution and evolution of climbing plants. In: F.E. Putz \& H.A. Mooney (eds.). The Biology of Vines. Cambridge, Cambridge University Press, pp. 3-49.

Gerwing, J.J., Schnitzer, S.A., Burnham, R.J., Bongers, F., Chave, J., Dewalt, S.J., Ewango, C.E.N., Foster, R., Kenfack, D., Martínez-Ramos, M., Parren, M., Parthasarathy, N., Pérez-Salicrup, D.R., Putz, F.E. \& Thomas, D.W. 2006. A standard protocol for liana censures. Biotropica 38: 256-261.

Giudice Neto, J., Pinto, M.M. \& Rossi, L. 2010. Plano de Manejo Integrado das Unidades de Conservação: Reserva Biológica e Estação Ecológica de Mogi Guaçu - SP. Instituto de Botânica, São Paulo.

Hegarty, E.E. 1991. Vine-host interactions. In: F.E. Putz \& H.A. Mooney (eds.). The Biology of Vines. Cambridge University Press, Cambridge, pp. 357-375.

Hora, R.C. \& Soares, J.J. 2002. Estrutura fitossociológica da comunidade de lianas em uma floresta estacional semidecidual na Fazenda Canchim, São Carlos, SP. Revista Brasileira de Botânica 25: 323-329.

Kim, A.C. 1996. Lianas da Mata Atlântica. Dissertação de Mestrado. Universidade Estadual de Campinas, Campinas.

Lima, H.C., Lima, M.P.M., Vaz, A.M.S.F. \& Pessoa, S.V.A. 1997. Trepadeiras da reserva ecológica de Macaé de Cima. In: H.C. Lima \& R.R. Guedes-Bruni (eds.). Serra de Macaé de Cima: Diversidade florística e conservação em Mata Atlântica. Jardim Botânico do Rio de Janeiro, Rio de Janeiro, pp. 75-87. 
Lombardi, J.A., Temponi, L.G. \& Leite, C.A. 1999. Mortality and diameter growth of lianas in a semideciduous forest fragment in Southeastern Brazil. Acta Botanica Brasilica 13: 159-165.

Magurran, A.E. 1996. Ecological diversity and its measurement. Chapman \& Hall, Londres.

Mamede, M.C.H. 2010. Banisteriopsis in Lista de Espécies da Flora do Brasil. Jardim Botânico do Rio de Janeiro. http://floradobrasil.jbrj.gov.br/2010/FB008803 (acesso em 23.03.2012).

Mantovani, W. \& Martins, F.R. 1988. Variações fenológicas das espécies do cerrado da Reserva Biológica de Mogi Guaçu, Estado de São Paulo. Revista Brasileira de Botânica 11: 101-112.

Matteucci, S.D. \& Colma, A. 1982. Metodologia para el estudio de la vegetacion. Organización de los Estados Americanos, Washington D.C.

Morellato, L.P.C. \& Leitão Filho, H.F. 1990. Estratégias fenológicas de espécies arbóreas em floresta mesófila na Serra do Japi, Jundiaí, São Paulo. Revista Brasileira de Biologia 50: 163-173.

Morellato, L.P.C. \& Leitão Filho, H.F. 1996. Reproductive phenology of climbers in a southeasten Brasilian forest. Biotropica 28: 180-191.

Morellato, L.P.C. \& Leitão Filho, H.F. 1998. Levantamento florístico da comunidade de trepadeiras de uma floresta semidecídua no sudeste do Brasil. Boletim do Museu Nacional 103: 1-15.

Morellato, L.P.C., Rodrigues, R.R., Leitão Filho, H.F. \& Joly, C.A. 1989. Estudo fenológico comparativo de espécies arbóreas de floresta de altitude e floresta mesófila semidecídua na Serra do Japi, Jundiaí, SP. Revista Brasileira de Botânica 12: 85-98.

Morellato, L.P.C., Rodrigues, R.R., Leitão Filho, H.F. \& Joly, C.A. 1990. Estratégias fenológicas de espécies arbóreas em floresta de altitude na Serra do Japi, Jundiaí, SP. Revista Brasileira de Biologia 50: 149-162.

Mueller-Dombois, D. \& Ellemberg, H. 1974. Aims and methods of vegetation ecology. John Willey, New York.

Oliveira, P.E.A.M. \& Moreira, A.G. 1992. Anemocoria em espécies de cerrado e mata de galeria de Brasília, DF. Revista Brasileira Botânica 15: 163-174.

Opler, P.A., Baker, H.G. \& Frankie, G.W. 1991. Seasonality of climber communities: a review and example from Costa Rica dry forest. In: F.E. Putz \& H.A. Mooney (eds.). The Biology of Vines. Cambridge, Cambridge University Press, pp. 337-391.

Penhalber, E.F. 1995. Fenologia, chuva de sementes e estabelecimento de plântulas em um trecho de mata em São Paulo, SP. Tese de Doutorado, Universidade de São Paulo, São Paulo.
Proctor, J., Anderson, J.M. \& Chai, P. 1983. Ecological studies in four constrasting rain forest in Gunung Mulu National Park. Journal of Ecology 71: 237-260.

Putz, F.E. 1984. The natural history of lianas on Barro Colorado Island, Panamá. Ecology 65: 1713-1724.

Putz F. E. \& Chai P. 1987. Ecological studies of lianas in Lambir National Park, Sarawak. Journal of Ecology 75: 523-531.

Putz, F.E. \& Mooney, H.A. 1991. The Biology of Vines. Cambridge University Press, Cambridge.

Rezende, A.A. \& Ranga, N.T. 2005. Lianas da Estação Ecológica do Noroeste Paulista, São José do Rio Preto/Mirassol, SP, Brasil. Acta Botanica Brasilica 19: 273-279.

Reys, P., Galetti, M., Morelatto, L.P.C. \& Sabino, J. 2005. Fenologia reprodutiva e disponibilidade de frutos de espécies arbóreas em mata ciliar no Rio Formoso, Mato Grosso do Sul. Biota Neotropica 5: 1-10.

Rodrigues, R.R. \& Leitão Filho, H.F. 2001. Matas ciliares: conservação e recuperação. Editora Edusp, São Paulo.

Rossi, L. 1994. Aflora arbórea-arbustiva da mata da reserva da Cidade Universitária “Armando de Salles Oliveira” (São Paulo, Brasil). Boletim do Instituto de Botânica 9: 1-105.

Rubim, P., Nascimento, H.E.M. \& Morelatto, L.P.C. 2010. Variações interanuais na fenologia de uma comunidade arbórea de floresta semidecídua no sudeste do Brasil. Acta Botanica Brasilica 24: 756-764.

Santos, K., Kinoshita, L.S. \& Rezende, A.A. 2009. Species composition of climbers in seasonal semideciduous forest fragments of Southeastern Brazil. Revista Biota Neotropica 9: 175-188.

Shepherd, G.J. 1995. Manual de usuário: Fitopac. Universidade Estadual de Campinas, Campinas.

Somner, G. 2009. Sapindaceae. In: M.G.L. Wanderley, G.J. Shepherd, T.S. Melhem, A.M. Giulietti \& S.A. Martins (eds.). Flora Fanerogâmica do Estado de São Paulo 6, Fapesp, São Paulo, pp. 195-256.

Tibiriçá, Y.J.A., Coelho, L.F.M. \& Moura, L.C. 2006. Florística de lianas em um fragmento de floresta estacional semidecidual, Parque Estadual de Vassununga, Santa Rita do Passa Quatro, SP, Brasil. Acta Botanica Brasilica 20: 339-346.

Udulutsch, R.G., Assis, M.A. \& Picchi, D.G. 2004. Florística de trepadeiras numa floresta estacional semidecídua, Rio Claro-Araras, Estado de São Paulo, Brasil. Revista Brasileira de Botanica 27: 125-134.

Venturi, S. 2000. Florística e fitossociologia do componente apoiante-escandente em uma floresta costeira subtropical. Dissertação de Mestrado. Universidade Federal do Rio Grande do Sul, Porto Alegre.

Villagra, B.L.P. \& Romaniuc Neto, S. 2010. Florística de trepadeiras no Parque Estadual das Fontes do Ipiranga, São Paulo, Brasil. Revista Brasileira de Biociências 8: $186-200$.

Whitmore, T.C. 1990. An introduction to tropical rain forests. Clarendon Press, Oxford. 